\title{
Ruolo della metformina nella prevenzione dell'osteoporosi
}

\author{
Simone Antonini ${ }^{1,2} \cdot$ Gherardo Mazziotti $^{1,2}$ \\ Accettato: 1 settembre 2021 / Pubblicato online: 4 novembre 2021 \\ (c) The Author(s), under exclusive licence to Springer Nature Switzerland AG 2021
}

Commento a:

Metformin use is associated with a lower risk of osteoporosis/vertebral fracture in Taiwanese patients with type 2 diabetes mellitus.

\section{C.-H. Tseng.}

Eur J Endocrinol (2021) 184(2):299-310

Il diabete mellito di tipo 2 (DMT2) e l'osteoporosi rappresentano entrambe patologie con crescente incidenza nella popolazione generale, costituendo spesso comorbidità che gravano sugli stessi gruppi di pazienti. Inoltre, il diabete mellito rappresenta una condizione predisponente a fragilità scheletrica, attraverso molteplici meccanismi che vanno ad alterare il processo di rimodellamento scheletrico con conseguente perdita di massa ossea, alterazione della microstruttura ossea e aumentato rischio di fratture da fragilità. In tale contesto di elevato rischio fratturativo, diventa importante per l'endocrinologo la scelta di farmaci anti-diabetici ad azione protettiva per lo scheletro.

In un recente studio, pubblicato da Tseng nel febbraio 2021 sullo European Journal of Endocrinology [1], si è evidenziato un ruolo protettivo della metformina sulla comparsa di osteoporosi e di fratture vertebrali con Hazard Ratio (HR) di 0,592 (95\% CI: 0,550-0,638), in un'ampia popolazione di 14611 pazienti con recente diagnosi di diabete mellito tipo 2 (DMT2) e senza pregressa storia di osteoporosi o fratture di fragilità. L'effetto protettivo della metformina era età-indipendente, veniva osservato in entrambi i sessi e risultava evidente già dopo 6 mesi di trattamento. Tali risultati convergono con quelli descritti dallo studio REDLINCIX del 2020 [2] su una coorte di donne sudamericane, che evidenziava il ruolo protettivo della terapia con metformina sia in pazienti con che senza DMT2.

\footnotetext{
$凶$ S. Antonini

simone.antonini@humanitas.it

1 Unità di Endocrinologia, Diabetologia e Andrologia Medica, IRCCS Istituto Clinico Humanitas, Rozzano, MI, Italia

2 Dipartimento di Scienze Biomediche, Humanitas University, Pieve Emanuele, MI, Italia
}

Qual è il meccanismo alla base del possibile effetto antifratturativo della metformina nei pazienti con diabete mellito tipo 2? In aggiunta ai noti effetti favorevoli sul metabolismo glucidico che indirettamente indurrebbero un miglioramento della salute scheletrica, alcuni studi sperimentali suggeriscono che il farmaco possa avere un'azione diretta sugli osteoblasti, favorendone la funzione e, quindi, migliorando possibilmente la disfunzione osteoblastica che è alla base della fragilità scheletrica nel paziente con diabete mellito [3]. Questi effetti pro-osteoblastici potrebbero spiegare anche l'azione protettiva della metformina sull'insorgenza di osteonecrosi della mandibola su un modello murino trattato con acido zoledronico [4]. Studi futuri consentiranno di chiarire se gli effetti protettivi della metformina possano potenziarsi in terapia di combinazione con farmaci, come le incretine, che hanno precedentemente dimostrato anch'essi un effetto anti-fratturativo [5].

\section{Bibliografia}

1. Tseng CH (2021) Metformin use is associated with a lower risk of osteoporosis/vertebral fracture in Taiwanese patients with type 2 diabetes mellitus. Eur J Endocrinol 184(2):299-310

2. Blümel JE, Arteaga E, Aedo S et al (2020) Metformin use is associated with a lower risk of osteoporosis in adult women independent of type 2 diabetes mellitus and obesity. REDLINC IX study. Gynecol Endocrinol 36(5):421-425

3. Jiating L, Buyun J, Yinchang Z (2019) Role of metformin on osteoblast differentiation in type 2 diabetes. BioMed Res Int 2019:9203934

4. Nakagawa T, Tsuka S, Aonuma F et al (2021) Effects of metformin on the prevention of bisphosphonate-related osteonecrosis of the jaw-like lesions in rats. J Prosthodont Res 65(2):219-224

5. Monami M, Dicembrini I, Antenore A, Mannucci E (2011) Dipeptidyl peptidase-4 inhibitors and bone fractures: a meta-analysis of randomized clinical trials. Diabetes Care 34(11):2474-2476

Nota della casa editrice Springer Nature rimane neutrale in riguardo alle rivendicazioni giurisdizionali nelle mappe pubblicate e nelle affiliazioni istituzionali. 